\title{
Non-invasive Determination of Aortic Mechanical Properties and Their Effects on Left Ventricular Function Following Endovascular Abdominal Aneurysm Repair
}

\author{
Alexander Gregory ${ }^{1,3} \cdot$ Marelise Kruger ${ }^{1} \cdot$ Neal Maher $^{1} \cdot$ Randy Moore $^{2} \cdot$ Gary Dobson $^{1}$
}

Received: 12 April 2018 / Accepted: 22 October 2018 / Published online: 2 November 2018

(c) The Author(s) 2018

\begin{abstract}
Background Increased pulse-wave velocity (PWV) entails elevated arterial stiffness and is associated with adverse cardiovascular outcomes. Previous studies have shown PWV increases following endovascular aneurysm repair (EVAR). Animal models suggest elevated PWV following endograft deployment occurs in conjunction with decreased aortic compliance and increased aortic impedance. This could lead to unwanted effects on left ventricular (LV) function. This study evaluates the early implications of EVAR on aortic mechanics and associated LV function.

Materials and methods Prospective observational study of elective EVAR for abdominal aneurysm. Transesophageal echocardiography was used to acquire LV flow and function, as well as images of the ascending aorta. Speckle tracking echocardiography (STE) software analysis allowed for determination of aortic volume change throughout the cardiac cycle. Aortic mechanics (including compliance, impedance and reflected wave analysis) and left-ventricular function (including cardiac output, hydraulic load and diastolic function) were measured pre- and post-endograft deployment.

Results Endograft deployment resulted in no significant increase in aortic impedance. It did alter the timing of reflected waves in the aorta, with a greater positive wave being reflected during systole. This increased the hydraulic load on the LV with corresponding statistically non-significant trends for decreasing cardiac output and LV diastolic function.

Discussion STE represents an emerging imaging modality for aortic biomechanical assessment. The increased PWV seen following EVAR may not reflect the same aortic biomechanical pathophysiology as in the non-surgical population with measures of increased arterial stiffness. The stiffer endograft does not appear to acutely increase aortic impedance, though it does alter the timing of reflected waves which may have negative effects on LV function.
\end{abstract}

Keywords Endovascular aneurysm repair - Aortic compliance - Aortic strain · Aortic impedance - Wave form analysis · Speckle tracking echocardiography

Alexander Gregory

alex.gregory@albertahealthservices.ca

Marelise Kruger

mkruger@footit.ca

Neal Maher

neal.maher@albertahealthservices.ca

Randy Moore

drrdmoore@gmail.com

Gary Dobson

gary.dobson@albertahealthservices.ca
1 Department of Anesthesiology, Perioperative and Pain Medicine, Cumming School of Medicine, University of Calgary, Calgary, AB, Canada

2 Division of Vascular Surgery, Peter Lougheed Hospital, Calgary, AB, Canada

3 Libin Cardiovascular Institute, Calgary, AB T2N 2T9, Canada 


\section{Introduction}

Elevated measures of arterial stiffness (central and peripheral) have been shown to negatively impact LV systolic and diastolic function, impair coronary blood flow, and have been identified as a risk factor for cardiovascular disease and mortality [1-9]. One possible mechanism for these findings is that increased arterial stiffness can create additional LV work while simultaneously making coronary perfusion less efficient. The hydraulic load (IE: work) encountered during LV ejection is directly increased when arterial stiffening (less compliance) results in higher aortic input impedance. The size and timing of reflected waves will also change in the setting of a stiff arterial tree, often with a larger positive reflected wave returning to the proximal aorta in systole instead of diastole. This alteration of reflected wave properties can indirectly add to the increased LV hydraulic load, while also causing diminished coronary perfusion.

Endovascular aneurysm repair (EVAR) is well-established and frequently used treatment modality for aortic pathology, including abdominal aortic aneurysms (AAA). Accordingly, much research has been generated on the local effects of the graft with respect to the mechanisms of endoleak, graft migration and aneurysm sac remodeling. There has also been interest in the arterial physiologic effects of placing artificial graft material into the aorta, as well as the potential implications on cardiac function. A dog model demonstrated that proximal thoracic aortic stenting results in increased overall aortic input impedance, ascending aorta characteristic impedance (Z0) and pulse-wave velocity (PWV). These alterations occurred in conjunction with decreased total arterial compliance, alterations in reflected wave properties and reduced LV output (measured as lower aortic flow) [10]. An increased PWV has subsequently been demonstrated in human patients following implantation of an EVAR endograft [11-16]. Although this suggests an endograft induced increase in aortic stiffness, a comprehensive analysis of the biomechanical impact of endograft implantation has not been possible due to the invasive measures that would be required.

Recent improvements in medical imaging, combined with the rapid growth in computing power, has resulted in an emergence of non- or minimally-invasive methods for performing more nuanced determinations of aortic mechanical properties. Ultrasound images of the aorta can be acquired with intraoperative transesophageal echocardiography (TEE). These images can then be analyzed with speckle tracking (STE), a validated method which is able to follow tissue motion and quantify position, strain, and velocity $[17,18]$. Though mostly used for quantifying ventricular function, this technology has been used to quantify aortic properties- including correlation with histologic and physical biomechanical testing of subsequently excised aortic tissue [19-21].

We report a technique using STE to assist in non-invasively determining the immediate effects of abdominal endograft deployment on aortic input impedance, ascending aortic compliance, systemic vascular resistance, reflected wave properties and LV function.

\section{Materials and Methods}

This study received approval from our institutional ethic committee. Participants were selected from adult (age $>18$ years) patients scheduled to have elective EVAR for AAA. Exclusion criteria included patient refusal, contraindication to trans-esophageal echocardiography, prior aortic surgery, aortic valve abnormality (stenosis, regurgitation or prosthesis), atrial fibrillation and severe aortic calcification ( $>2 / 3$ circumference). After being identified and enrolled in the study demographic, medical information and current medications were collected. All procedures were performed under general anesthesia. Pre-implantation data was acquired once the patient was in a plane of anesthesia with stable hemodynamics. Post-implantation data was acquired in the operating theatre, shortly after endograft deployment when the patient had stable hemodynamics.

\subsection{Data-Collection and Analysis}

\subsubsection{Echocardiography}

Trans-esophageal echocardiogram (TEE) was performed in each patient using a GE Vivid 7 machine (General Electric, Fairfield, Connecticut, USA). The following views were obtained (Fig. 1):

1. Mid-esophageal 4-chamber view.

2. Tissue-Doppler imaging (TDI) and pulsed-wave Doppler (PWD) of the lateral and septal mitral valve annuli.

3. PWD of the trans-mitral flow.

4. Mid-esophageal long axis view of the aortic valve, including the aortic root and proximal ascending aorta

5. Deep-transgastric view of the aortic valve with PWD of the left ventricular outflow tract (LVOT), just proximal to the aortic valve.

\subsubsection{Speckle-Tracking Echocardiography}

Analysis of the TEE images were performed post-acquisition offline using a PC workstation and EchoPac, Version 7.2.0 software (General Electric, Fairfield, Connecticut, USA). 

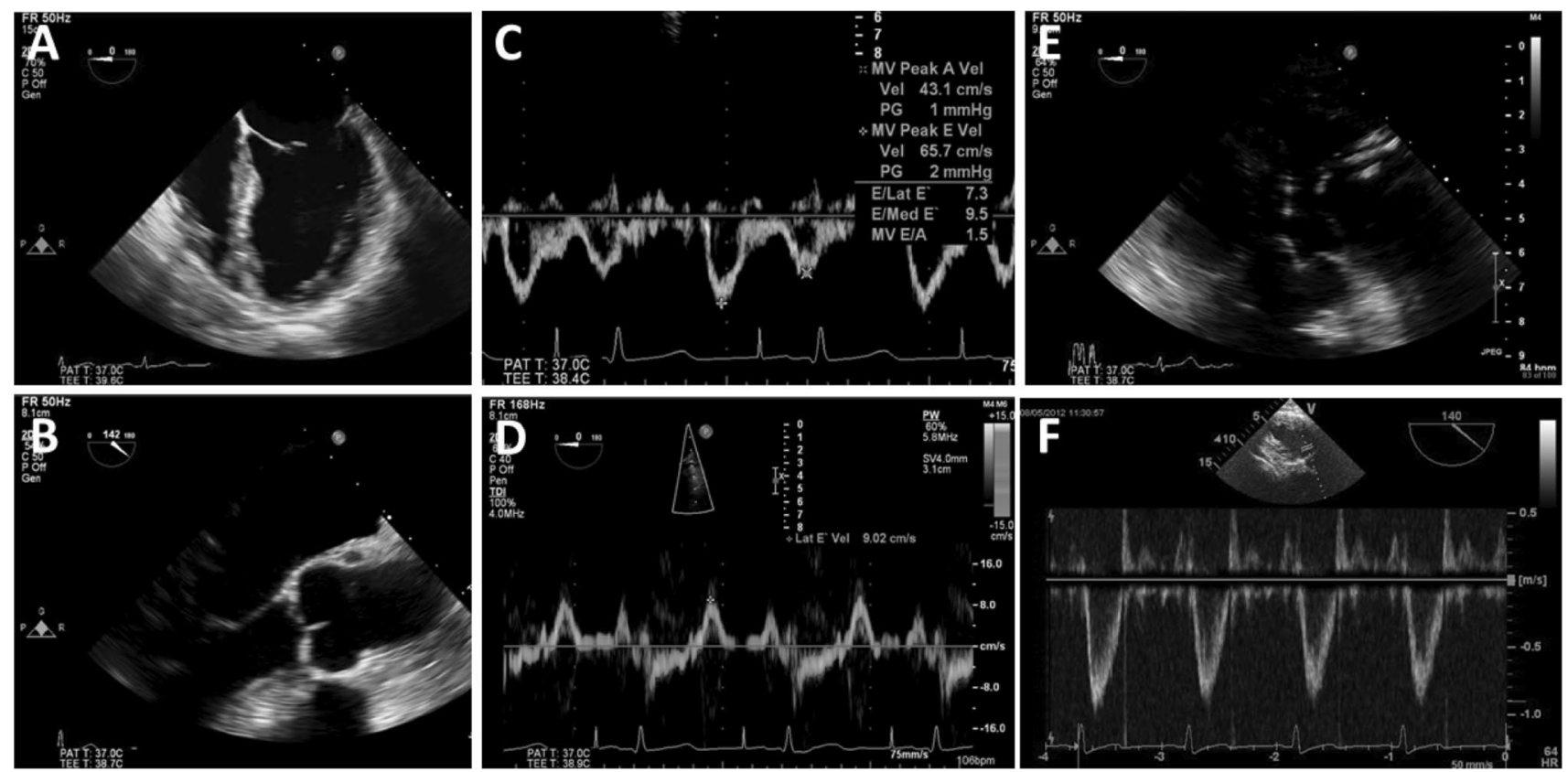

Fig. 1 Images demonstrating the standard views and Doppler quantification acquired from each patient before and after EVAR procedure. a Mid-esophageal 4-chamber view. b Mid-esophageal aortic valve long axis view. c Trans-mitral pulse wave Doppler flow. d Tis-

The value for peak mitral annulus TDI velocity in early diastole was obtained for both the septal and lateral annuli (E'sept and E'lat) at the same times peak trans-mitral flow velocity in early diastole (E) using PWD.

Measurements of proximal aortic dimensions were taken from the standard TEE mid-esophageal aortic long-axis view

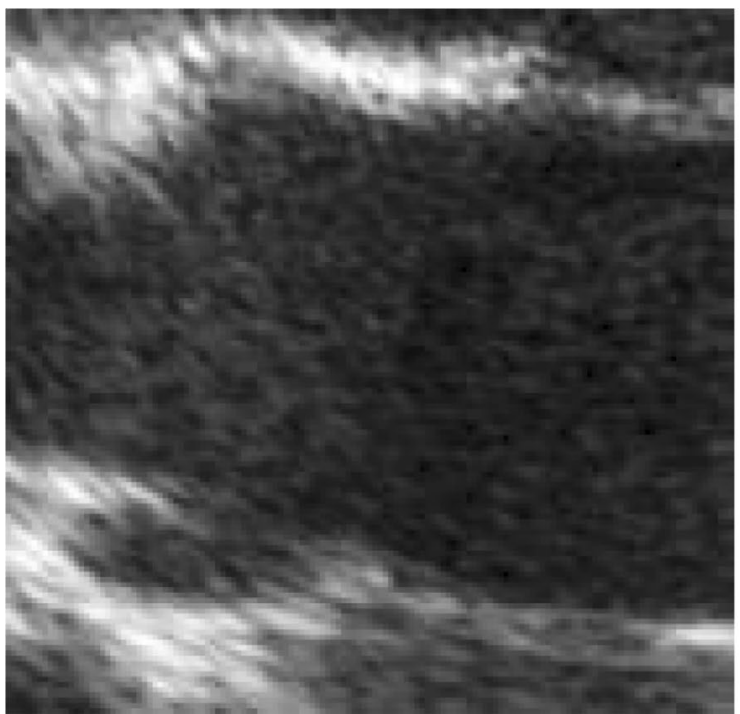

Fig. 2 Caliper measurements of the ascending aorta used to calculate compliance. Taken from the mid-esophageal long axis view of the aorta at end-systole. Al posterior length of segment 1 (aortic root), sue Doppler imaging of the basal septal and basal lateral LV walls. e Deep transgastric aortic valve/LVOT view. f LVOT pulse wave Doppler flow

using the caliper function (Fig. 2). The aorta was divided into 2 segments. The first segment was the aortic root while the second segment was the proximal ascending aorta. The anterior and posterior lengths of the aortic root were measured as 2 straight lines spanning, but not including, the walls of the Sinus of Valsava from the aortic valve to

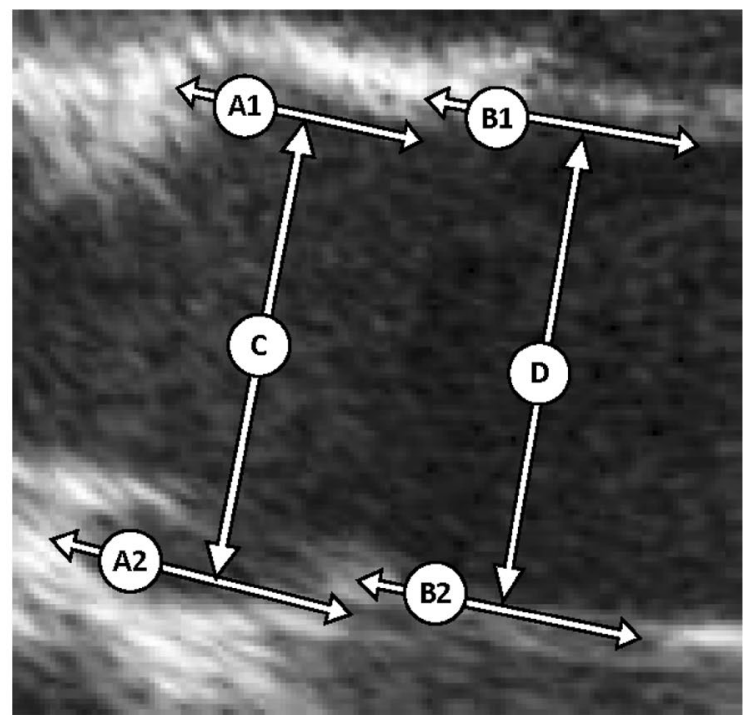

$A 2$ anterior length of segment $1, B 1$ posterior length of segment 2 (ascending aorta), B2 anterior length of segment 2, $C$ segment 1 diameter, $D$ segment 2 diameter. See Methods section for details 
the sinotubular junction. The second segment was measured as 2 straight lines along the anterior and posterior aortic walls, from the sinotubular junction extending $2 \mathrm{~cm}$ into the ascending aorta. The lengths of the second segment were shortened in the event of poor image quality or if the boundary of the image prevented a full $2 \mathrm{~cm}$ segment. The anterior and posterior segment lengths were averaged to obtain a single length for our calculation of aortic volume. The diameters of each segment were taken inner edge to inner edge, at the midpoint of the segment. The diameter of the LVOT was also measured from this view. All measurements were taken at end-systole.

Speckle tracking imaging (STI) analysis was performed using the same EchoPac software. In the mid- esophageal four-chamber view the endocardial border of the left ventricle (LV) was traced. Appropriate tissue tracking was confirmed with the program's own quality control feature, as well as visually by the investigators by observing a full cine loop to ensure the ventricular walls were tracked throughout the cardiac cycle. The STI-derived peak values for longitudinal tissue velocity, longitudinal strain rate and radial strain rate of the basal-septal and basal-lateral walls were measured in early diastole (Fig. 3).

STI analysis was further applied to the ascending aorta using the EchoPac software's pre-set for two- chamber LV images using a method we have previously described [4]. This method includes adjusting the software's pre-set spatial and timing filters to the lowest level. The walls of the aortic root and ascending aorta were traced from the aortic valve annulus to the most distal portion of the ascending aorta that was visible on the acquired images. Proper aortic tissue tracking was confirmed in the same fashion as mentioned above. The region of interest (ROI) was made as narrow as possible to best conform to the width of the aorta. The ROI borders were further adjusted so that the software's pre-set labels for identifying LV segments matched the aortic segments described above. The ROI for the LV basal segments spanned the aortic root while the LV mid segments spanned the proximal $2 \mathrm{~cm}$ of ascending aorta (Fig. 4). The LV apical segments did not track any aortic tissue and were not

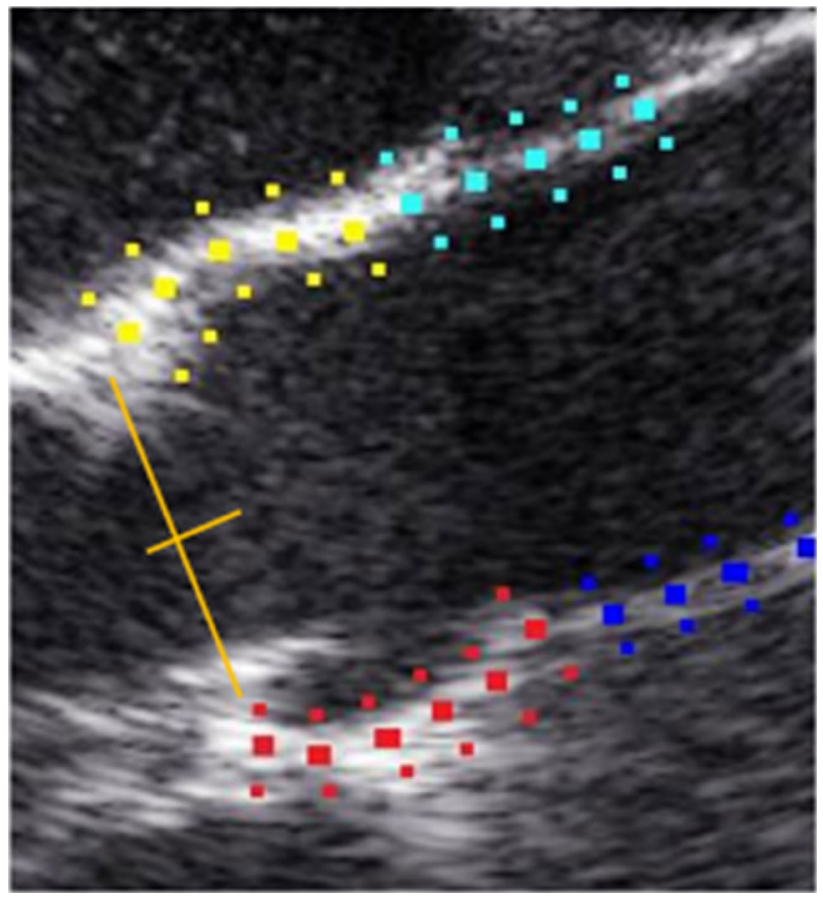

Fig. 4 The aortic root and proximal ascending aorta are traced using speckle tracking imaging software. The region of interest was adjusted so that the width incorporated the aortic wall. The segments were adjusted to include the posterior root (yellow), anterior root (red), posterior ascending aorta (cyan) and proximal ascending aorta (blue)

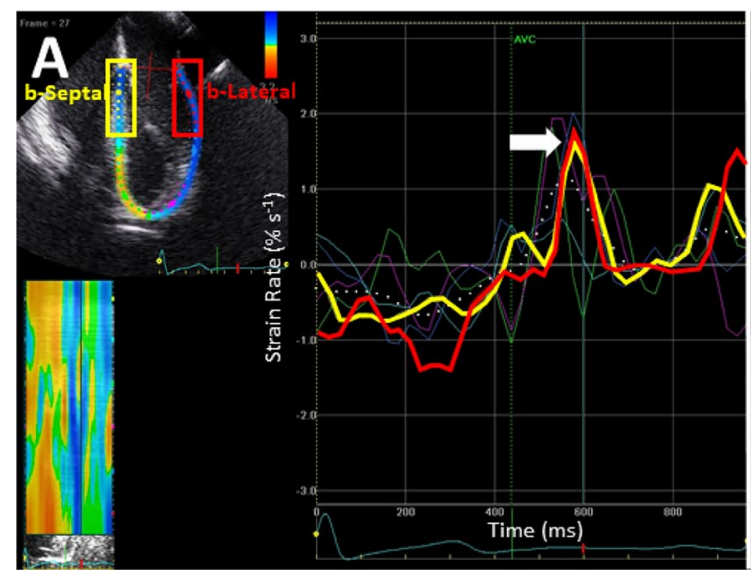

Fig. 3 Speckle tracking imaging of the LV. Peak longitudinal velocity, peak longitudinal strain rate (Fig. 3a) and peak radial strain rate (Fig. 3b) were taken in early diastole (E'). The basal septal wall is

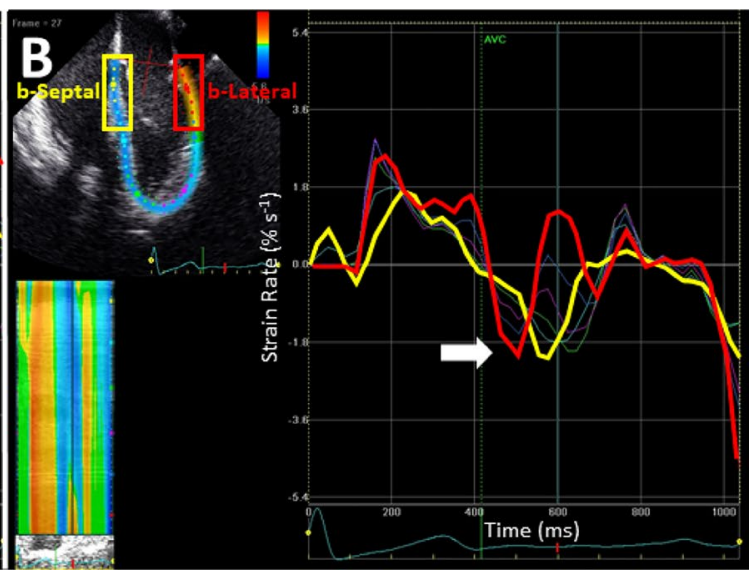

depicted in yellow, the basal lateral wall is depicted in red and the peak values are identified with a white arrow 
included in our analysis. Radial velocity and longitudinal strain rates were taken for the anterior and posterior walls of the aortic root and ascending aorta (Fig. 5). The graphs produced by the EchoPac software were converted into digital form using Digitize-It (version 1.5.8b, Bormisoft, Braunschweig, Germany) and saved as a text file.

\subsubsection{Arterial Pressure Acquisition and Aortic Pressure Derivation}

A right radial artery catheter placed prior to the induction of anesthesia. The catheter was connected to the transducer via a short length of pressure tubing $(12 \mathrm{~cm})$ to optimize the resonant frequency of the system. The filter of the arterial pressure channel on the Datex/Ohmeda S5 monitor was set to a frequency of $44 \mathrm{~Hz}$ and the analogue waveforms (radial artery pressure wave and the ECG) were acquired simultaneously through the data-out port at $1000 \mathrm{~Hz}$ using an analogue-digital converter (National Instruments, Austin, Texas, USA) and LabVIEW (National Instruments, Austin, Texas, USA). A pop-test was performed prior to every recording in order to calculate the system's resonant frequency and dampening coefficient. Ten to twelve pressure waves were averaged from the peak of the QRS complex of the ECG. The arterial pressure wave was then calibrated using the systolic and diastolic pressure recorded at the time of the acquisition. The pressure wave was then corrected for the resonance characteristics of the system and converted to an aortic pressure waveform using a previously validated transfer function [22].

\subsubsection{Calculation of Aortic Parameters}

Off-line analysis was performed using Maple (version 18.02, MapleSoft, Waterloo, Ontario, Canada). The aortic pressure wave was used to calculate the rate of pressure change $(\mathrm{dP} / \mathrm{dt})$ during the cardiac cycle. The STI based graphs of aortic radial velocity and longitudinal strain rate were used to calculate aortic volume and its rate of change during the cardiac cycle $(\mathrm{dV} / \mathrm{dt})$. All signals were filtered using a Butterworth, 4-element filter with a cut-off frequency of $16 \mathrm{~Hz}$. The minor differences in the duration of the $\mathrm{dP} / \mathrm{dt}$ and $\mathrm{dV} / \mathrm{dt}$ waves were corrected through interpolation of the $\mathrm{dV} / \mathrm{dt}$ waveforms. The ECG- gating from the TEE machine and anesthesia monitor were not identical resulting in a timing shift of the graphs of $\mathrm{dP} / \mathrm{dt}$ and $\mathrm{dV} /$ dt. Therefore, we aligned the two graphs at the point where both $\mathrm{dP} / \mathrm{dt}$ and $\mathrm{dV} / \mathrm{dt}$ were initially positive. The interactions of pressure and volume change were used to generate pressure-volume loops for each patient, both before and after stent implantation (Fig. 6). We then produced graphs displaying total proximal aortic compliance (combined aortic root + ascending aorta) over the entire cardiac cycle. To calculate proximal aortic systolic compliance, the start of systole was initially identified by considering the time point in the cardiac cycle where $\mathrm{dP} / \mathrm{dt}$ first
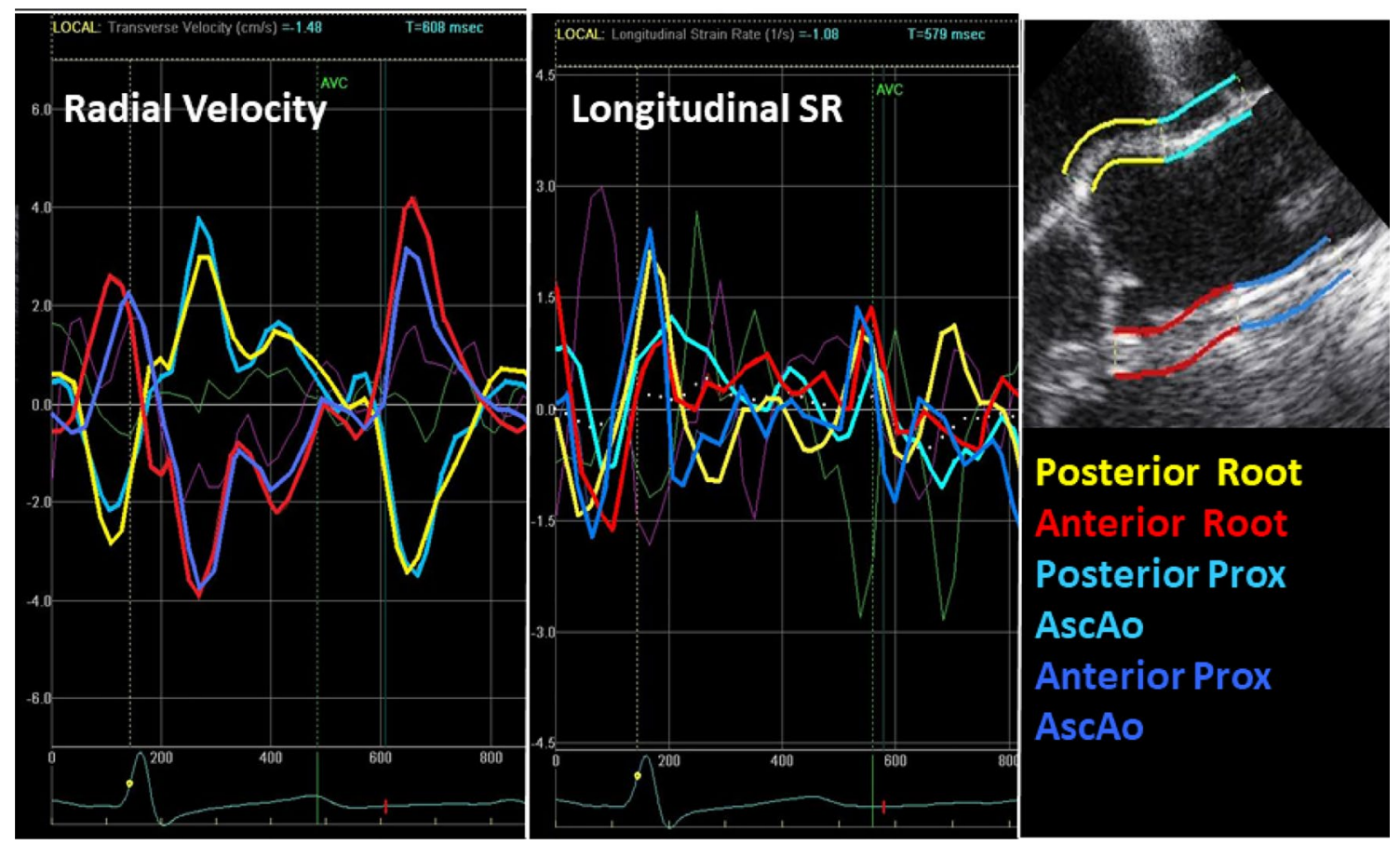

Fig. 5 Speckle tracking imaging analysis of the aortic root and proximal ascending aorta. Region of interest was adjusted as described in the text and Fig. 4. Radial velocity and longitudinal strain rates were taken throughout the cardiac cycle in order to measure aortic volume change 
Fig. 6 By combining STI-based aortic volume change with invasively measured arterial pressure pressure-volume loops were generated for each patient before and after deployment of the EVAR endograft. Arrows depict the direction of the loops during the cardiac cycle

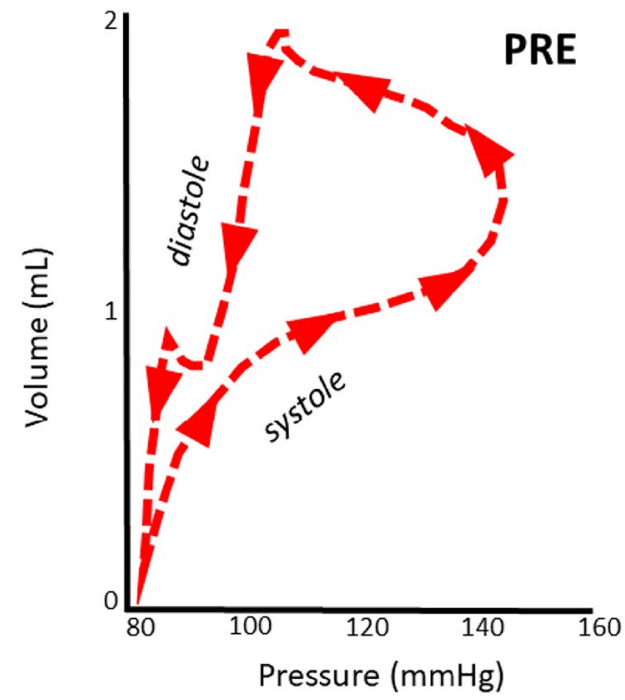

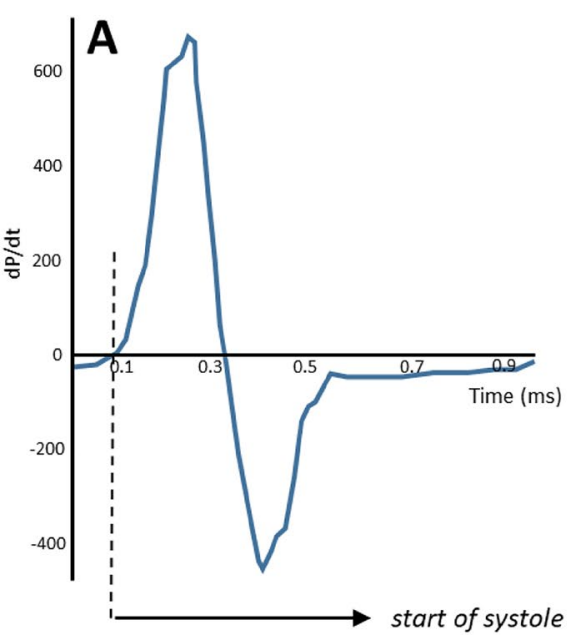

Fig. 7 The determination of proximal aortic systolic compliance was completed in a 2 -step process. The start of systole was determined by identifying the time point where $\mathrm{dP} / \mathrm{dt}$ first became positive (Fig. 7a). This time value was then used on the compliance curve to locate the start of systole (Fig. 7b). A consistent finding was an asymptote in this area. The mean compliance in the period from the first postasymptote peak until the beginning of the nadir was used to calculate proximal aortic systolic compliance (CAortaSyst) in our analysis. This period is represented by the shaded box becomes positive (Fig. 7a). The value of this time point was then used on the generated compliance versus time curve to identify the approximate beginning of systole. An asymptote near the start of systole and a flat nadir at the end of systole were consistent findings in all the study subjects (Fig. 7b). There was high variability in both the data values near the asymptote and the length of the nadir. Therefore, to obtain consistent measurements between patients, the first post-asymptote peak on the compliance curve in systole was chosen as the starting point and the beginning of the nadir as the end-point for measuring early systolic aortic compliance. Thus, proximal aortic systolic compliance (CAortaSyst) was defined as the average compliance over this period (Fig. 7b).

The LVOT flow acquired from the intra-operative TEE using PWD was digitized (DigitizeIt version 1.5.8b, Bormisoft, Braunschweig, Germany) at $2000 \mathrm{~Hz}$, resampled at $1000 \mathrm{~Hz}$ and filtered. Stroke volume (SV) and cardiac index (CI) were calculated:

$\mathrm{SV}=\pi \times$ LVOT $_{\text {radius }}^{2} \times$ LVOT velocity time integral

$\mathrm{CI}=\mathrm{SV} \times \mathrm{HR} / \mathrm{BSA}$ 
By combining LVOT flow and central aortic pressure, aortic input impedance was plotted across the frequency spectrum up to $15 \mathrm{~Hz}$. The zero modulus was taken as systemic vascular resistance (SVR). The aortic characteristic impedance $(\mathrm{ZO})$ was calculated from the average impedance in the $5-15 \mathrm{~Hz}$ frequencies, excluding any values that were more than 2 standard deviations above the average. The left ventricular pulsatile power, including mean and oscillatory power, was calculated from the pressure and flow waves using methodology previously described [23]. Additional use of the aortic pressure included wave form analysis allowing identification of the forward (incident) and backward (reflected) components (Fig. 8). This was done according to previously accepted methods [24-27]. We calculated the total forward, total backward, systolic forward and systolic backward waves. The reflection coefficient $(\mathrm{PB} / \mathrm{PF})$ was determined by the total reflected wave divided by the total forward wave.

\subsection{Statistical Analysis}

A sample size was calculated that would allow for comparison of post-EVAR changes in both LV diastolic function and aortic characteristic impedance. We used data from a previous study which measured characteristic impedance in both normotensive and hypertensive middle-aged patients, similar to our patient population. Their results showed a mean $\mathrm{Z} 0$ of 166 dynes s cm${ }^{-5}$ with a standard deviation of 69 dynes s cm${ }^{-5}$ [28]. Eighteen patients would allow for a detection of a post-implantation increase in impedance of $40 \%$ with $80 \%$ power and a $p$ value $<0.05$ [24]. A $40 \%$ relative change in impedance was felt to be clinically significant based on a similar change described by Dobson et al. [10]. This assertion is supported by studies showing that a 20-40\% difference in valvuloarterial impedance impacts the clinical outcomes in patients with aortic valve stenosis [29].
With regards to diastolic function, STI values in middle-aged patients with mild or moderate diastolic dysfunction, the average longitudinal early diastolic strain rate was $1.15 \% / \mathrm{s}$ with a standard deviation of $0.26 \% / \mathrm{s}$ [30]. Twentyone patients would allow for detecting a $20 \%$ decrease in diastolic function following EVAR with a power of $80 \%$ and a $p$ value $<0.05$ [31]. This change is likely clinically relevant based on the findings of Tsioufis et al., where a $20 \%$ difference in diastolic function was observed between normotensive and hypertensive patients [32].

All statistical analysis was performed using Analyze-It software version 3.90.7 (Analyze-It Software Ltd, Leeds, UK). Descriptive statistics were used for demographic and intra-operative data. The values for $\mathrm{LV}$ diastolic function and all measures of aortic function were analyzed for changes following endograft implantation using a Student's $T$ test.

\section{Results}

Twenty-one patients were included in the study. Three of the patients had incomplete data sets due to equipment failure or inadequate TEE images, therefore a total of 18 patients' data was analyzed. The demographic information and medical information is summarized in Table 1. Intraoperative hemodynamic details and endograft information is listed in Table 2. There was a near-significant trend towards higher systolic blood pressure after the EVAR procedure increasing from a mean (SD) of 109.5 (17.1) $\mathrm{mmHg}$ to 119.0 (20.3) $\mathrm{mmHg}(p=0.06)$. There was no significant difference after EVAR in diastolic blood pressure $(p=0.59)$, mean arterial pressure $(p=0.35)$, heart rate $(p=0.19)$, and a trend towards reduced cardiac index $(p=0.11)$.

Pre-operatively the peak systolic mean (SD) STE measures of aortic longitudinal strain rates were $1.36(0.80) \% / \mathrm{s}$ for the anterior segments and $1.57(0.73) \% / \mathrm{s}$ for the posterior segments while the STE aortic radial velocities were
Fig. 8 Example from one of our study subjects of our wave form analysis using the aortic pressure wave (solid; red) allowing identification of the forward (hashed; blue) and backward (hashed-dot; pink) components. A difference can be seen in the size and timing of the reflected wave in a pre-EVAR compared to $\mathbf{b}$ post-EVAR
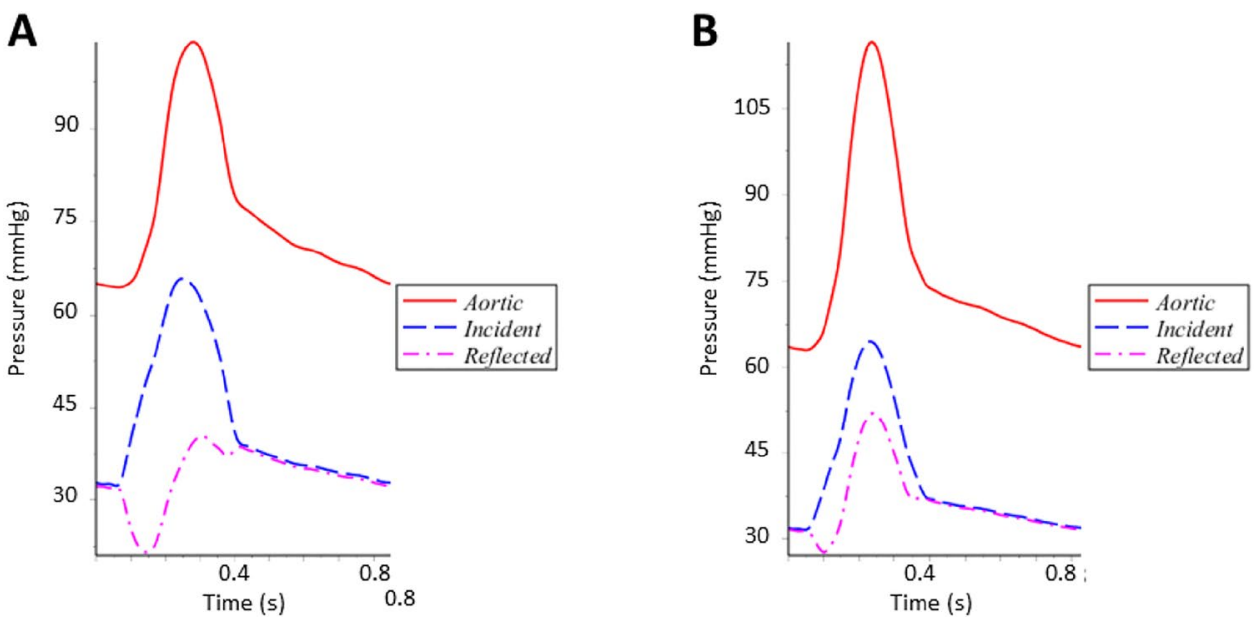
Table 1 Patient demographics, comorbidities and pre-operative medications

\begin{tabular}{ll}
\hline Age (years) & $71(7)$ \\
\hline Gender $(\%$ male) & 89 \\
Height $(\mathrm{cm})$ & $175(8)$ \\
Weight $(\mathrm{kg})$ & $90(19)$ \\
BMI $\left(\mathrm{kg} / \mathrm{m}^{2}\right)$ & $29.3(5.1)$ \\
BSA $\left(\mathrm{m}^{2}\right)$ & $2.08(0.24)$ \\
Comorbidities $(\%)$ & \\
Hypertension & 83 \\
Smoking & 28 \\
Chronic lung disease & 17 \\
Coronary artery disease & 61 \\
Congestive heart failure & 6 \\
Cerebrovascular disease & 0 \\
Diabetes mellitus & 17 \\
Chronic renal failure & 0 \\
Dyslipidemia & 67 \\
Pre-op medications (\%) & \\
Beta-blockers & 44 \\
Calcium-channel blockers & 22 \\
ACEi/ARB & 78 \\
Diuretics & 28 \\
Statins & 67 \\
Nitrates & 6 \\
\hline
\end{tabular}

Values are expressed as mean (SD) or as percentage where indicated $A C E i$ angiotensin-converting enzyme inhibitors, $A R B$ angiotensin-II receptor blockers, $B M I$ body mass index, $B S A$ body surface area

$3.30(1.12) \mathrm{cm} / \mathrm{s}$ for the anterior segments and $3.02(0.95)$ $\mathrm{cm} / \mathrm{s}$ for the posterior segments. Post-operatively peak systolic aortic longitudinal strain rates were $1.69(1.10) \% / \mathrm{s}$ for the anterior segments and $1.78(1.00) \% / \mathrm{s}$ for the posterior segments while the aortic radial velocities were $3.52(0.99)$ $\mathrm{cm} / \mathrm{s}$ for the anterior segments and $3.22(1.18) \mathrm{cm} / \mathrm{s}$ for the posterior segments.

Full results of the comparative analysis of aortic and left ventricular diastolic function post-EVAR are included in Tables 3 and 4. Following deployment of the endovascular stent graft proximal aortic systolic compliance $(p=0.60)$ and calculated SVR $(p=0.21)$ were not significantly altered by EVAR endograft deployment while the aortic characteristic impedance $(\mathrm{Z} 0)$ had a borderline significant postEVAR decrease from a mean (SD) of 133.8 (63.6) dynes s/ $\mathrm{cm}^{5}$ to 102.3 (77.5) dynes $\mathrm{s} / \mathrm{cm}^{5}(p=0.06)$. The magnitude of forward and reflected waves did not change significantly, however the reflection coefficient increased from a mean (SD) of $0.85(0.08)$ to $0.88(0.07)(p=0.03)$. This was due to a change in the timing of positive reflected waves, with the systolic component increasing significantly $(p=0.002)$. The left ventricular pulsatile power (hydraulic load) increased
Table 2 Intra-operative clinical and procedural data

\begin{tabular}{|c|c|}
\hline \multicolumn{2}{|c|}{ Pre-EVAR hemodynamic parameters } \\
\hline $\mathrm{SBP}(\mathrm{mmHg})$ & $110(17)$ \\
\hline $\mathrm{DBP}(\mathrm{mmHg})$ & $59(6)$ \\
\hline MAP (mmHg) & $79(12)$ \\
\hline HR (beats $\min ^{-1}$ ) & $59(9)$ \\
\hline $\operatorname{SVI}\left(\mathrm{mL} / \mathrm{m}^{2}\right)$ & $29(7)$ \\
\hline $\mathrm{CI}\left(\mathrm{L} / \mathrm{min} / \mathrm{m}^{2}\right)$ & $1.7(0.5)$ \\
\hline \multicolumn{2}{|c|}{ Post-EVAR hemodynamic parameters } \\
\hline $\mathrm{SBP}(\mathrm{mmHg})$ & $119(20)$ \\
\hline $\mathrm{DBP}(\mathrm{mmHg})$ & $60(10)$ \\
\hline MAP (mmHg) & $82(16)$ \\
\hline HR (beats $\min ^{-1}$ ) & $61(8)$ \\
\hline $\mathrm{SVI}\left(\mathrm{mL} / \mathrm{m}^{2}\right)$ & $31(6)$ \\
\hline $\mathrm{CI}\left(\mathrm{L} / \mathrm{min} / \mathrm{m}^{2}\right)$ & $1.9(0.4)$ \\
\hline \multicolumn{2}{|c|}{ Endograft used [number (\%)] } \\
\hline Anaconda & $11(61)$ \\
\hline Cook low profile & $1(6)$ \\
\hline Excluder & $1(6)$ \\
\hline Gore TAG & $3(17)$ \\
\hline Medtronic & $1(6)$ \\
\hline OLB 32 & $1(6)$ \\
\hline
\end{tabular}

Values are expressed as mean (SD) or as number (\%) where indicated $C I$ cardiac index, $D B P$ diastolic blood pressure, EVAR endovascular aneurysm repair, $H R$ heart rate, $M A P$ mean arterial blood pressure, $S B P$ systolic blood pressure, $S V I$ stroke volume index

from a mean (SD) of 160.1 (108.3) $\mathrm{mW}$ to 206.3 (93.8) $\mathrm{mW}(p=0.05)$. There was a non-significant trend towards increased LV diastolic impairment post-EVAR, in both TDI and STI measured parameters (Table 4).

\section{Discussion}

Our results show that immediately after deployment of an EVAR endograft there was no increase in aortic characteristic impedance, ascending aortic compliance or systemic vascular resistance. The positive reflected wave remained the same size, however the timing of its reflection was altered, with a greater portion now returning during systole. We found that the LV experienced an increased pulsatile load, though the LV stroke volume and cardiac index were not affected. Finally, there may have been a small impairment of LV diastolic function, but not to a great enough extent to meet statistical significance.

As the aorta stiffens pulse waves originating in the heart conduct to the periphery with increasing velocity. As a result, pulse wave velocity (PWV) can be used as an indirect measure of the relative stiffness of the arterial system. It has been frequently used in clinical studies quantifying 
Table 3 Aortic properties, LV pulse power and wave analysis results

\begin{tabular}{llll}
\hline & PRE & POST & $p$ value \\
\hline SVR $($ dynes s/cm & \\
$\mathrm{Z}_{0}\left(\right.$ dynes s $\left./ \mathrm{cm}^{5}\right)$ & $1694(513)$ & $1574(497)$ & 0.21 \\
Mean syst comp $\left(\mathrm{mL} /\right.$ dynes $\left./ \mathrm{m}^{2}\right)$ & $134(64)$ & $102(78)$ & 0.06 \\
Pulse power $(\mathrm{mW})$ & $4.14 \mathrm{E}^{-5}\left(2.16 \mathrm{E}^{-5}\right)$ & $4.49 \mathrm{E}^{-5}\left(3.25 \mathrm{E}^{-5}\right)$ & 0.60 \\
Total FWD $\left(\right.$ dynes $\left./ \mathrm{cm}^{2}\right)$ & $160(108)$ & $206(93)$ & 0.05 \\
Systolic FWD $\left(\right.$ dynes $\left./ \mathrm{cm}^{2}\right)$ & $5.56 \mathrm{E} 4(9.58 \mathrm{E} 3)$ & $5.66 \mathrm{E} 4(1.38 \mathrm{E} 4)$ & 0.73 \\
Total reflected $\left(\right.$ dynes $\left./ \mathrm{cm}^{2}\right)$ & $2.55 \mathrm{E} 4(6.81 \mathrm{E} 3)$ & $2.76 \mathrm{E} 4(7.89 \mathrm{E} 3)$ & 0.20 \\
Systolic reflected $\left(\right.$ dynes $\left./ \mathrm{cm}^{2}\right)$ & $4.72 \mathrm{E} 4(9.22 \mathrm{E} 3)$ & $4.96 \mathrm{E} 4(1.13 \mathrm{E} 4)$ & 0.24 \\
Reflect coeff & $1.74 \mathrm{E} 4(4.45 \mathrm{E} 3)$ & $2.09 \mathrm{E} 4(4.62 \mathrm{E} 3)$ & 0.002 \\
\hline
\end{tabular}

Values are expressed as mean (SD)

Mean Syst Comp mean ascending aorta systolic compliance, Reflect Coeff wave reflection coefficient, Systolic FWD systolic forward wave, SVR systemic vascular resistance, Total FWD total forward wave, $Z_{0}$ aortic characteristic impedance

\begin{tabular}{lrrr}
\hline & \multicolumn{1}{l}{ Pre } & \multicolumn{1}{l}{ Post } & $p$ value \\
\hline b-Lateral E' TDI (cm/s) & $8.21(1.93)$ & $7.71(2.46)$ & 0.17 \\
b-Septal E' TDI (cm/s) & $7.62(1.80)$ & $6.85(2.30)$ & 0.10 \\
b-Lateral E/E' & $9.05(2.71)$ & $9.78(4.21)$ & 0.33 \\
b-Septal E/E' & $9.80(3.29)$ & $11.04(5.40)$ & 0.19 \\
b-Lateral STI longitudinal velocity (cm/s) & $-6.49(2.18)$ & $-6.18(1.96)$ & 0.51 \\
b-Septal STI longitudinal velocity (cm/s) & $-5.65(1.85)$ & $-5.30(1.66)$ & 0.15 \\
b-Lateral STI Longitudinal SR (\%/s) & $1.05(0.56)$ & $1.05(0.45)$ & 0.98 \\
b-Septal STI Longitudinal SR (\%/s) & $0.88(0.47)$ & $0.78(0.31)$ & 0.46 \\
b-Lateral STI radial SR (\%/s) & $-3.65(2.00)$ & $-2.94(1.66)$ & 0.29 \\
b-Septal STI radial SR (\%/s) & $-2.22(1.13)$ & $-2.29(1.75)$ & 0.85 \\
\hline
\end{tabular}

Values are expressed as mean (SD)

$b$-Septal LV basal septal wall, b-Lateral LV basal lateral wall, SR strain rate, STI speckle tracking imaging, TDI tissue Doppler imaging changes in arterial stiffness due to aging or disease states. More importantly, increasing arterial stiffness as detected by elevated PWV has been linked to subclinical target organ disease and impaired cardiovascular health, including increased mortality [5-9]. The potential value of PWV as a clinical tool for improving long-term cardiovascular patient care has led to its inclusion in both the American Heart Association and European Society of Cardiologists' most recent guidelines on the management of arterial hypertension and aortic disease [33, 34].

The endografts used in EVAR procedures are made with material that is stiffer than the native aorta, even in the setting of atherosclerotic or aneurysmal disease. Therefore, it is not surprising that an increased PWV measured across the EVAR site has been a consistently reported finding. Lantelme et al. assessed 50 patients following either open or endovascular AAA repair. After a median follow-up interval of 47 days they demonstrated an increase in mean PWV from a baseline of 11.3 to $12.3 \mathrm{~m} / \mathrm{s}$, or approximately $10 \%$ $(p=0.001)$ [13]. Of note, the hemodynamic alterations were different between the surgical graft prosthesis and the endograft, suggesting device material cannot be ignored. PostEVAR increase in PWV was confirmed by Kadoglou, et al. with a $25 \%$ increase in mean PWV $(13.11 \mathrm{~m} / \mathrm{s}$ to $16.41 \mathrm{~m} / \mathrm{s})$ after 6 months [11]. The post-op intervals, population demographics and surgical specifics were not identical in each study, which may explain the difference in relative PWV change. Importantly, the finding of increased PWV postEVAR was consistent and both authors suggest that this elevation may have clinical implications given the clear association of elevated PWV and cardiovascular outcomes in non-surgical populations. Their concern is further supported by the results of subsequent studies where an increased post-EVAR PWV was also associated with higher levels of inflammatory markers, increased LV mass, and LV diastolic dysfunction $[12,15,16]$. In one study, increased LV mass was detected as early as one week following an EVAR procedure [16]. 
Studies in animal models shed light onto the implications of increased PWV with regards to concurrent changes in aortic mechanical properties and possible mechanisms for the negative LV effects observed in humans. Using a canine model Dobson et al. implanted an endograft in the thoracic aorta [10]. There was a significant elevation in PWV postdeployment (418 $\mathrm{cm} \mathrm{s}^{-1}$ vs. $\left.755 \mathrm{~cm} \mathrm{~s}^{-1}, p<0.05\right)$, a finding consistent with the EVAR patient studies mentioned above. This change occurred alongside other changes in the ascending aortic mechanical properties. Significant post-stent increases occurred in proximal aortic impedance $\left(\mathrm{Z} 0=0.250 \mathrm{mmHg} \mathrm{mL}^{-1} \mathrm{~s}^{-1}\right.$ vs. $0.414 \mathrm{mmHg} \mathrm{mL}^{-1} \mathrm{~s}^{-1}$, $p<0.05$ ). Additionally, total arterial compliance decreased $\left(0.446 \mathrm{~mL} \mathrm{mmHg}^{-1}\right.$ vs. $\left.0.276 \mathrm{~mL} \mathrm{mmHg}^{-1}, p<0.05\right)$ alongside a near- significant increase in SVR $\left(5.8 \mathrm{mmHg} \mathrm{mL}^{-1} \mathrm{~s}^{-1}\right.$ vs. $7.7 \mathrm{mmHg} \mathrm{mL}^{-1} \mathrm{~s}^{-1}, p=0.06$ ). There was also an alteration of reflected wave forms, with the reflected wave coefficient becoming dominated by a large negative reflected wave ( 0.09 vs $-0.49, p<0.05)$. Due to endograft-aorta impedance mismatch one would expect to see larger positive and negative reflected waves, from the proximal and distal ends of the graft respectively which would typically cancel each other. The authors suggest the change in wave co-efficient in their study was due to increased proximal aortic stiffness suppressing the positive reflected wave, unmasking the negative wave. Another interesting finding was a measurable decrease in aortic flow (IE cardiac output) which neared statistical significance ( $0.89 \mathrm{~L} \mathrm{~min}^{-1}$ vs. $0.66 \mathrm{~L} \mathrm{~min}^{-1}, p=0.09$ ), possibly as a result of the described changes in the aortic properties causing an increased LV hydraulic load. An alteration of aortic input impedance by thoracic grafting was similarly found in Mekkaoui et al. using a porcine model [35]. Of particular note, these changes resulted in an impairment of the natural diastolic augmentation of blood flow to the coronary and supra-aortic arteries. The findings of these papers suggest that (1) increased PWV may reflect other concurrent changes in aortic mechanics, including proximal characteristic impedance, (2) the net effect of these changes may have a deleterious effect on coronary blood flow, LV hydraulic load and cardiac output and (3) these results provide possible mechanistic explanations for the findings in post-EVAR human studies.

Previous studies in human subjects following EVAR had failed to demonstrate any "upstream" alteration in aortic stiffness [13, 36]. But these studies did not assess all of the aspects of aortic mechanical behaviour, nor did they measure aortic stiffness in the same fashion as the animal studies. This methodological discordance could easily explain the discrepant findings. Our study did not have such limitations. Even though our study methods closely approximated those in the animal studies, we did not find the changes in ascending aortic compliance or characteristic impedance identified by Dobson and Mekkaoui. An obvious explanation is that these changes simply do not occur in humans. Failed translation of animal models to human clinical studies is not uncommon. Other possible explanations do exist. Our patients, unlike the animal studies, had endografts deployed more distally in the aorta (abdomen vs thoracic) which may be too remote to induce changes in the ascending aorta. Alternatively, inhaled volatile anesthetic's suppression of the sympathetic nervous system may have counteracted the proposed mechanism of sympathetic activation for the changes in local aortic mechanics following endograft implantation [37-39]. Our measured SVR was lower post-EVAR, likely reflecting the cumulative effect of general anesthesia on decreased sympathetic/vascular tone at the level of the small arteries and arterioles (considered to be the largest contributors to SVR) [40]. It should be noted that neither set of animals in the comparison studies were anesthetized in a similar fashion to our patients (IE general anesthesia with inhaled volatile anesthetics).

Reflected waves occur whenever a point of impedance mismatch occurs along the arterial tree. A transition point to higher or lower impedance results in positive and negative reflected waves respectively. There are naturally occurring reflection points in the normal human arterial system, a negative wave reflects near the origins of the abdominal vessels while a positive one originates near the femoral arteries. As the arterial tree stiffens due to aging and vascular disease the size and timing of the positive reflected wave may change. Instead of providing diastolic blood flow augmentation, the wave arrives during systole. This results in added hydraulic load on the left ventricle, impaired coronary blood flow, LV diastolic dysfunction and contribution to long term adverse cardiovascular outcomes [41-43]. Stiffer endograft material also creates an impedance mismatch which may affect wave reflection properties. Our data suggests that although the absolute size of the positive reflected wave did not change, the timing was altered with an increased systolic component. This systolic reflected wave augmentation likely explains the increased fractional pulse pressure and trend towards higher systolic blood pressure, with no corresponding change in mean or diastolic pressures. It may also explain the increased LV hydraulic load and trend towards lower cardiac output despite the marginally decreased aortic impedance. The mechanism for previously reported PWV associated changes in LV mass and diastolic function following EVAR may in fact be a result of endograft induced changes in reflected wave properties, not increased aortic impedance.

Diastolic function has been shown to deteriorate in the setting of increases in measures of arterial stiffness (including PWV), as well as fractional pulse pressure and reflected waves [2, 32, 44-49]. If implantation of an abdominal endograft in an EVAR procedure alters any or all of these aortic properties, there may be clinical implications on left ventricular diastolic function. Several of 
our measures of LV diastolic function suggest relaxation is impaired following EVAR, though they did not meet statistical significance. More time may be required for diastolic function to be affected following EVAR. Takeda et al. found no change in diastolic function in the setting of increased PWV post-EVAR. However, at a 1 year follow-up over half the patients had deterioration in exercise tolerance and reduced diastolic function [15]. Therefore, although we did see statistically significant changes in diastolic function, there is both substrate (IE immediate increase in LV hydraulic load) and evidence to suggest that given enough time or a larger sample size we may see diastolic dysfunction in these patients.

\subsection{Limitations}

Our parameters, though comprehensive, were only measured immediately following endograft deployment. Therefore, we do not know how any of these might progress over time. Do those that become altered, such as reflected wave properties and LV hydraulic load, remain the same, become more prominent, or eventually normalize? Do factors that did not change, such as proximal aortic compliance and diastolic function change over time? During intra-operative data collection our patients were under general anesthesia which impacts LV preload, afterload and sympathetic tone, potentially affecting our results. We were unable to include measurements of PWV concurrently with our other parameters. This limits the ability to put our findings into context with those from studies where increased PWV was measured post-EVAR. Given the ubiquity of elevated PWV following EVAR it is reasonable to assume we would have had a similar increase as well. We are not able to comment on the effects of different graft manufacturers or materials on the parameters measured because a majority of our patients had the same endograft implanted. Previous studies have suggested that differences between graft materials affect measures of aortic stiffness [12, 13, 37]. Finally, we have no control group with which to compare. One study that did include matched controls found that although EVAR grafts acutely increased PWV and reflected waves, these changes remained stable over time. The control group had gradual increases in PWV and reflected waves over time, and eventually the two groups were similar [14]. This suggests that the immediate changes in aortic stiffness, wave reflections and cardiac function following EVAR may just accelerate changes to the cardiovascular system which would have occurred over time in a non-surgical cohort. Whether it is detrimental to EVAR patients to have these changes occur acutely and sooner than would naturally occur is a question that requires further study.

\section{Conclusion/Future Directions}

Primarily, our study demonstrates that speckle tracking echocardiography represents a promising tool to non-invasively quantify aortic biomechanics in a real-time clinical setting.

In the setting of EVAR for abdominal aneurysms, measures of aortic stiffness that directly affect LV afterload, such as aortic impedance, ascending aortic compliance and SVR do not immediately increase following endograft implantation. The graft does appear to create an impedance mismatch which results in changes to the timing of positive reflected waves resulting in a greater systolic component. This timing shift is associated with an increased LV hydraulic load, which showed a trend towards decreased cardiac output and impaired LV diastolic relaxation [23].

To better describe the natural progression of aortic and cardiac changes following EVAR further studies should focus on the following: (1) Measure the corresponding changes in proximal aortic impedance, ascending aortic compliance, pulse wave analysis, PWV, LV hydraulic load, and LV systolic/diastolic function immediately following the procedure (IE after effects of general anesthesia are gone), then again at mid- and long-term intervals. (2) Examine these same parameters after placing stents in more proximal locations such as the thoracic aorta, aortic arch and ascending aorta. (3) Perform a similar comprehensive analysis with a variety of graft materials and designs (both surgical and endovascular) to determine inter-graft differences. These additional studies would allow for a more thorough description of the changes that occur after endovascular graft procedures, the clinical implications of these changes, and further explore ways to minimize any potential negative effects with different graft designs or materials.

Author contributions All authors were actively involved in the conception, design, implementation, and data collection for the study. All authors met to discuss the findings of the study with respect to manuscript preparation prior to it being written. AG was the principal author of the manuscript's first draft, produced the figures and tables, and edited subsequent revisions. MK reviewed/edited the manuscript first draft and made significant editorial contributions to the final version which has been submitted. NM reviewed/edited the manuscript first draft and made significant editorial contributions to the final version which has been submitted. RM reviewed/edited the manuscript first draft and made significant editorial contributions to the final version which has been submitted. GD was the co-author of the manuscript's first draft. He was the principal author involved in data analysis and helped produce many of the figures/tables. He also reviewed/edited the original manuscript and made significant editorial contributions to the final version which has been submitted.

\section{Compliance with ethical standards}

Disclosures None of the listed authors has any financial or personal relationships with other people or organizations that could potentially/ inappropriately influence their work and conclusions with respect to 
this study or the submitted manuscript. This research did not receive any specific grant from funding agencies in the public, commercial, or not-for-profit sectors.

Open Access This article is distributed under the terms of the Creative Commons Attribution 4.0 International License (http://creativeco mmons.org/licenses/by/4.0/), which permits unrestricted use, distribution, and reproduction in any medium, provided you give appropriate credit to the original author(s) and the source, provide a link to the Creative Commons license, and indicate if changes were made.

\section{References}

1. Al-Mallah, M. H., Nasir, K., Katz, R., et al. (2014). Relation of thoracic aortic distensibility to left ventricular area (from the multi-ethnic study of atherosclerosis [MESA]). American Journal of Cardiology, 113(1), 178-182.

2. Kawaguchi, M., Hay, I., Fetics, B., et al. (2003). Combined ventricular systolic and arterial stiffening in patients with heart failure and preserved ejection fraction: implications for systolic and diastolic reserve limitations. Circulation, 107(5), 714-720.

3. Ohtsuka, S., Kakihana, M., Watanabe, H., et al. (1994). Chronically decreased aortic distensibility causes deterioration of coronary perfusion during increased left ventricular contraction. Journal of the American College of Cardiology, 24(5), 1406-1414.

4. Gregory, A. J., \& Dobson, G. (2013). Proximal aortic compliance and diastolic function assessed by speckle tracking imaging. Canadian Journal of Anaesthesia, 60(7), 667-674.

5. Cooper, L. L., Rong, J., Benjamin, E. J., et al. (2015). Components of hemodynamic load and cardiovascular events: The Framingham Heart Study. Circulation, 131(4), 354-361.

6. Redheuil, A., Wu, C. O., Kachenoura, N., et al. (2014). Proximal aortic distensibility is an independent predictor of all-cause mortality and incident CV events: The MESA study. Journal of the American College of Cardiology, 64(24), 2619-2629.

7. Laurent, S., Boutouyrie, P., Asmar, R., et al. (2001). Aortic stiffness is an independent predictor of all-cause and cardiovascular mortality in hypertensive patients. Hypertension, 37(5), 1236-1241.

8. Mitchell, G. F., Hwang, S. J., Vasan, R. S., et al. (2010). Arterial stiffness and cardiovascular events: The Framingham Heart Study. Circulation, 121(4), 505-511.

9. Vlachopoulos, C., Aznaouridis, K., \& Stefanadis, C. (2010). Prediction of cardiovascular events and all- cause mortality with arterial stiffness: A systematic review and meta-analysis. Journal of the American College of Cardiology, 55(13), 1318-1327.

10. Dobson, G., Flewitt, J., Tyberg, J. V., et al. (2006). Endografting of the descending thoracic aorta increases ascending aortic input impedance and attenuates pressure transmission in dogs. European Journal of Vascular and Endovascular Surgery, 32(2), 129-135.

11. Kadoglou, N. P., Moulakakis, K. G., Papadakis, J., et al. (2012). Changes in aortic pulse wave velocity of patients undergoing endovascular repair of abdominal aortic aneurysms. $J$ Endovasc Ther., 19(5), 661-666.

12. Kadoglou, N. P., Moulakakis, K. G., Papadakis, J., et al. (2014). Differential effects of stent-graft fabrics on arterial stiffness in patients undergoing endovascular aneurysm repair. $J$ Endovasc Ther., 21(6), 850-858.

13. Lantelme, P., Dzudie, A., Miloln, H., et al. (2009). Effect of abdominal aortic grafts on aortic stiffness and central hemodynamics. Journal of Hypertension, 27(6), 1268-1276.
14. Lee, C. W., Sung, S. H., Chen, C. K., et al. (2013). Measures of carotid-femoral pulse wave velocity and augmentation index are not reliable in patients with abdominal aortic aneurysm. Journal of Hypertension, 31(9), 1853-1860.

15. Takeda, Y., Sakata, Y., Ohtani, T., et al. (2014). Endovascular aortic repair increases vascular stiffness and alters cardiac structure and function. Circulation Journal, 78(2), 322-328.

16. Taylor, J. (2011). The endovascular stent graft raises vascular stiffness and changes cardiac structure within a very short time. European Heart Journal, 32(14), 1693-1694.

17. Geyer, H., Caracciolo, G., Abe, H., et al. (2010). Assessment of myocardial mechanics using speckle tracking echocardiography: Fundamentals and clinical applications. Journal of the American Society of Echocardiography, 23, 351-369.

18. Amundsen, B. H., Helle-Valle, T., Edvardsen, T., et al. (2006). Noninvasive myocardial strain measurement by speckle tracking echocardiography: Validation against sonomicrometry and tagged magnetic resonance imaging. Journal of the American College of Cardiology, 47, 789-793.

19. Petrini, J., Yousry, M., Rickenlund, A., et al. (2010). The feasibility of velocity vector imaging by transesophageal echocardiography for assessment of elastic properties of the descending aorta in aortic valve disease. Journal of the American Society of Echocardiography, 23, 985-992.

20. Alreshidan, M., Shahmansouri, N., Chung, J., et al. (2017). Obtaining the biomechanical behavior of ascending aortic aneurysm via the use of novel speckle tracking echocardiography. Journal of Thoracic and Cardiovascular Surgery, 153, 781-788.

21. Emmott, A., Alzahrani, H., Alreishidan, M., et al. (2018). Transesophageal echocardiographic strain imaging predicts aortic biomechanics: Beyond diameter. The Journal of Thoracic and Cardiovascular Surgery (in press).

22. Karamanoglu, M., O'Rourke, M. F., Avolio, A. P., et al. (1993). An analysis of the relationship between central aortic and peripheral upper limb pressure waves in man. European Heart Journal, 14(2), 160-167.

23. Milnor, W. R. (1988). Hemodynamics (2nd ed.). Baltimore, Maryland: Williams \& Wilkins.

24. Laxminarayan, S. (1979). The calculation of forward and backward waves in the arterial system. Medical \& Biological Engineering \& Computing, 17(1), 130.

25. Merillon, J. P., Lebras, Y., Chastre, J., et al. (1983). Forward and backward waves in the arterial system, their relationship to pressure waves form. European Heart Journal, 4(Suppl G), 13-20.

26. Segers, P., Rietzschel, E. R., De Buyzere, M. L., et al. (2007). Noninvasive (input) impedance, pulse wave velocity, and wave reflection in healthy middle-aged men and women. Hypertension, 49(6), 1248-1255.

27. Westerhof, N., Sipkema, P., van den Bos, G. C., et al. (1972). Forward and backward waves in the arterial system. Cardiovascular Research, 6(6), 648-656.

28. Ting, C. T., Brin, K. P., Lin, S. J., et al. (1986). Arterial hemodynamics in human hypertension. Journal of Clinical Investigation, 78(6), 1462-1471.

29. Jang, J. Y., Seo, J. S., Sun, B. J., et al. (2016). Impact of valvuloarterial impedance on concentric remodeling in aortic stenosis and its regression after valve replacement. Journal of Cardiovascular Ultrasound, 24(3), 201-207.

30. Kim, H., Shin, H. W., Son, J., et al. (2011). Two-dimensional strain or strain rate findings in mild to moderate diastolic dysfunction with preserved ejection fraction. Heart and Vessels, 26(1), 39-45.

31. Rohrig, B., du Prel, J. B., Wachtlin, D., et al. (2010). Sample size calculation in clinical trials: Part 13 of a series on evaluation of scientific publications. Deutsches Ärzteblatt International, 107(31-32), 552-556. 
32. Tsioufis, C., Chatzis, D., Dimitriadis, K., et al. (2005). Left ventricular diastolic dysfunction is accompanied by increased aortic stiffness in the early stages of essential hypertension: A TDI approach. Journal of Hypertension, 23(9), 1745-1750.

33. Whelton, P. K., Carey, R. M., Aronow, W. S., et al. (2018). ACC/ AHA/AAPA/ABC/ACPM/AGS/APhA/ASH/ASPC/NMA/PCNA guideline for the prevention, detection, evaluation, and management of high blood pressure in adults: A report of the american college of cardiology/american heart association task force on clinical practice guidelines. Journal of the American College of Cardiology, 71(19), e127-e248.

34. Williams, B., Mancia, G., Spiering, W., et al. (2018). ESC/ESH guidelines for the management of arterial hypertension. European Heart Journal

35. Mekkaoui, C., Rolland, P. H., Friggi, A., et al. (2003). Pressureflow loops and instantaneous input impedance in the thoracic aorta: Another way to assess the effect of aortic bypass graft implantation on myocardial, brain, and subdiaphragmatic perfusion. Journal of Thoracic and Cardiovascular Surgery, 125(3), 699-710.

36. van Herwaarden, J. A., Muhs, B. E., Vincken, K. L., et al. (2006). Aortic compliance following EVAR and the influence of different endografts: Determination using dynamic MRA. Journal of Endovascular Therapy, 13(3), 406-414.

37. Rolland, P. H., Charifi, A. B., Verrier, C., et al. (1999). Hemodynamics and wall mechanics after stent placement in swine iliac arteries: Comparative results from six stent designs. Radiology, 213(1), 229-246.

38. Stone, D. N., \& Dujardin, J. P. (1985). Pressure dependence of the canine aortic characteristic impedance and the effects of alterations in smooth muscle activity. Medical \& Biological Engineering \& Computing, 23(4), 324-328.

39. Neukirchen, M., \& Kienbaum, P. (2008). Sympathetic nervous system: Evaluation and importance for clinical general anesthesia. Anesthesiology, 109(6), 1113-1131.
40. Tyberg, J. V., Bouwmeester, J. C., Parker, K. H., et al. (2014). The case for the reservoir-wave approach. International Journal of Cardiology, 172, 299-306.

41. Westerhof, B. E., Guelen, J., Westerhof, N., et al. (2006). Quantification of wave reflection in the human aorta from pressure alone: A proof of principle. Hypertension, 48(4), 595-601.

42. Narayan, O., Davies, J. E., Hughes, A. D., et al. (2015). Central aortic reservoir-wave analysis improves prediction of cardiovascular events in elderly hypertensives. Hypertension, 65(3), 629-635.

43. Mahfouz, R. A. (2013). Relation of coronary flow reserve and diastolic function to fractional pulse pressure in hypertensive patients. Echocardiography., 30(9), 1084-1090.

44. Blacher, J., Asmar, R., Djane, S., et al. (1999). Aortic pulse wave velocity as a marker of cardiovascular risk in hypertensive patients. Hypertension, 33(5), 1111-1117.

45. Borlaug, B. A., Melenovsky, V., Redfield, M. M., et al. (2007). Impact of arterial load and loading sequence on left ventricular tissue velocities in humans. Journal of the American College of Cardiology, 50(16), 1570-1577.

46. Russo, C., Jin, Z., Palmieri, V., et al. (2012). Arterial stiffness and wave reflection: Sex differences and relationship with left ventricular diastolic function. Hypertension, 60(2), 362-368.

47. Suh, S. Y., Kim, E. J., Choi, C. U., et al. (2009). Aortic upper wall tissue Doppler image velocity: Relation to aortic elasticity and left ventricular diastolic function. Echocardiography., 26(9), 1069-1074.

48. Triantafyllidi, H., Rizos, I., Rallidis, L., et al. (2010). Aortic distensibility associates with increased ascending thoracic aorta diameter and left ventricular diastolic dysfunction in patients with coronary artery ectasia. Heart and Vessels, 25(3), 187-194.

49. Pirracchio, R., Cholley, B., De Hert, S., et al. (2007). Diastolic heart failure in anaesthesia and critical care. British Journal of Anaesthesia, 98(6), 707-721. 